\title{
Prevention of Cerebral Herniation by Decompressive Craniecto- my in a Child with Acute Peritentorial Subdural Hematoma and Brain Edema: A Case Report
}

\author{
Vaner Köksal* \\ Department of Neurosurgery, Recep Tayyip Erdoğan University, Turkey
}

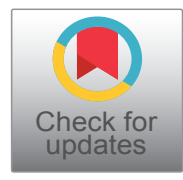

*Corresponding author: Vaner Köksal, Department of Neurosurgery, Recep Tayyip Erdoğan University, Research and Training Hospital, 53020 Rize, Turkey, Tel: +90-(464)-2130491

\begin{abstract}
Objective: Acute peritentorial subdural hematoma (APS$\mathrm{DH}$ ) is an extremely rare intracranial bleeding, which generally occurs due to a trauma. And, It usually regresses spontaneously without the need for a surgical intervention. However, it is difficult to determine the surgical method when it is life-threatening.
\end{abstract}

Case report: A 15-year-old boy was brought to the hospital after a crash with a car whilst on a motorcycle. No significant intracranial bleeding was detected following an isolated severe head trauma; however, Glasgow Coma Score was 8 due to the serious cerebral edema. Uncal herniation and anisocoria were detected 6 hours later. The brain tomography scan was repeated and a hematoma was detected on the surface of the tentorium. Stopping the herniation was necessary. The patient underwent decompressive temporo-parietal craniectomy duraplasty and extensive in order to access the base of the brain.

Results: Herniation was stopped and peritentorial blood spontaneously reduced. APSDH usually does not exert a significant mass effect alone. If it co-exist with severe cerebral edema, it may be cause to cerebral herniation. The extensive cranial decompression surgery was performed on the skull base. Although it was the indirect surgical approach, Decreased peritentorial bleeding volume was detected following the surgery. This result is not the expected. But, There is proof that how flexible and strong pulsations of his the brain tissue in a young man with intracranial hypertension.

Conclusions: No direct surgical intervention could be performed in the presence of a life-threatening by bleeding in a hard-to-reach area. Although direct surgery for hematoma is not performed, the primary aim is to urgently prevent cerebral herniation. We were thinking that this method for a bleeding in peritentorial localization can be applied.

\section{Keywords}

Decompressive craniotomy, Head trauma, Peritentorial, Subdural hemorrhage, Uncal herniation

\section{Introduction}

Acute subdural bleedings developing subsequent to head traumas are frequently observed on the outer surfaces of brain convexities, but may very rarely be observed on the upper surface of tentorium (peritentorial area) as well $[1,2]$. The literature data demonstrate that it is particularly observed as a result of post-partum trauma in difficult births in the newborn. It may be difficult to determine these bleedings on the tentorial surface via routine axial computed tomographic sections. Coronal and sagittal plain images other than the classic sections should also be obtained. However, despite these new imaging techniques, bleedings of this localization are not frequently encountered. Furthermore, it may be misdiagnosed due to co-existing bleedings and a complicated clinical course in patients with severe head trauma. The main problem is to determine the appropriate approach in the presence of a life-threatening bleeding in the peritentorial localization that causes uncal herniation.

\section{Case Report}

A 15-year-old boy was brought to the hospital due to a crash with a car while on a motorcycle. He was unconscious, agitated and localized the pain. The Glasgow Coma Score was 7-8. No anisocoria was determined; his 

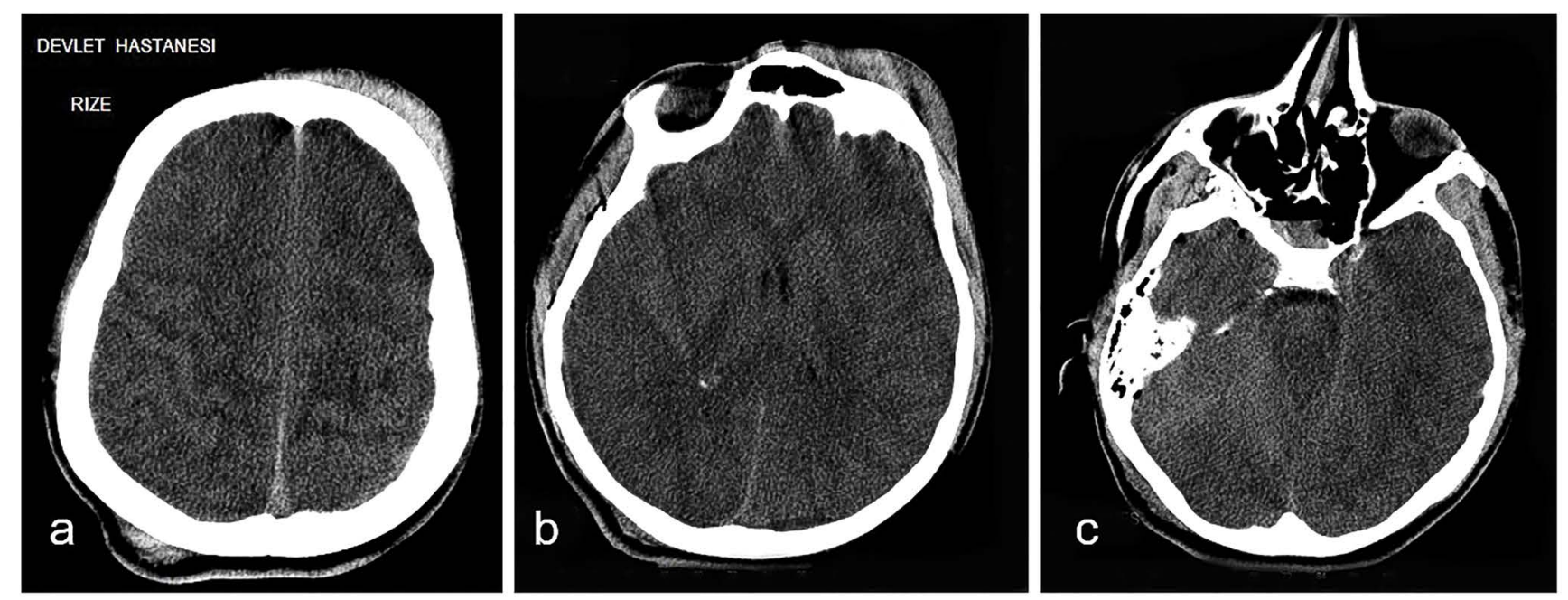

Figure 1: The first axial CT images of the patient in emergency department.

No sign of a significant haemorrhage. Sections bring to mind that diffuse axonal injury with petechial contusions.

respiration was irregular and there was hyperventilation. The first brain computed tomography (CT) showed diffuse cerebral edema and frontal subcortical and tentorial subarachnoid traumatic bleedings from one place to another (Figure 1a, Figure $1 \mathrm{~b}$ and Figure 1c). A comminuted femur fracture was also observed. The patient was taken to the intensive care unit with the diagnosis of severe head trauma; he was intubated and deep sleep was induced. The patient had also femoral shaft fracture due to multiple trauma together with traumatic brain injury. Anti-aggregation treatment (enoxoparin sodium $40 \mathrm{mg}$ ) was started immediately for the prevention of venous thromboembolism. Traction and fixation were performed for prevention of fat embolism on the femoral bone with fracture. A $3 \mathrm{~mm}$-sized anisocoria was observed in the right eye of the patients 6 hours later. The brain CT was repeated. The outer surface of the right hemisphere was clear, and a hematoma with insignificant borders lying beneath the tentorium was detected in 2 sections close to the tentorial area (Figure $2 \mathrm{a}$, Figure $2 \mathrm{~b}$, Figure $2 \mathrm{c}$ and Figure $2 \mathrm{~d}$ ). Furthermore, a right to left shift of 8-9 $\mathrm{mm}$ was detected in the midline (Figure 2d).

The clinical situation was considered to be cerebral herniation due to anisocoria, hyperventilation despite the suppression of the sleep-inducing drug, and bradycardia from time to time, diffuse brain edema in the brain $\mathrm{CT}$ and the midline shift. However, the localization of bleeding was a very rare one; therefore, the proper approach was decided to primarily stop the herniation. Due to the difficulty in direct access to the hematoma and the increased pressure in the right cerebral hemisphere, decompressive craniectomy was performed on the right hemisphere in an emergency fashion in order to de-stress the temporal base. Duroplasty was performed via fascia lata and the cranial piece was left inside the leg where the fascia lata had been obtained. The herniation was stopped after the operation. His respiration pattern improved and anisocoria regressed.
Neurological wellness was confirmed by the control brain CT images. The cisternae that had been observed to have been obliterated around the brain stem re-appeared and the midline shift regressed to $2-3 \mathrm{~mm}$ (Figure $2 \mathrm{e}$, Figure $2 \mathrm{f}$ and Figure $2 \mathrm{~g}$ ). A very surprising outcome was also observed. Although the hematoma was not accessed directly, the volume of peritentorial hematoma was observed to have decreased (Figure $2 \mathrm{~h}$ ).

The sleep induction of the patient was stopped on the post-op $2^{\text {nd }}$ day. His gases of the blood deteriorated abruptly when his spontaneous movements began on the $3^{\text {rd }}$ day. Suddenly, the patient began symptoms of respiratory insufficiency with petechial rashes on his body. Blood gases were deteriorating rapidly. Pulmonary failure developed in a short time, and despite the ventilatory support and medical treatments (corticosteroids), the blood gases did not turn to normal. Pulmonary embolism was detected in lung tomography and the patient passed away (exitus). Prognosis was very poor and fast. This bad result could not be prevented. In the light of current findings, pulmonary fat embolism was considered.

\section{Discussion}

The most fatal aspect of head traumas is the formation of cerebral edema that increases the intracranial pressure, which develops secondary to the brain damage. If the clinical presentation of cerebral herniation is realized at an early stage and treated emergently, functional complete recovery may be possible. Otherwise, death is inevitable $[1,3,4]$. Traumatic cerebral bleedings are rarely localized on the upper surface of the tentorium. However, there are possible reasons for cerebral bleeding to form in that localization. The first of them is the development of an inter-hemispheric bleeding; the second is the rupture of the bridge veins with the rupture in the tentorium, and the third is the contusion in the cerebral parenchyma [1,2]. Continuous bleeding of all contusions within the occipital cortex may lead 

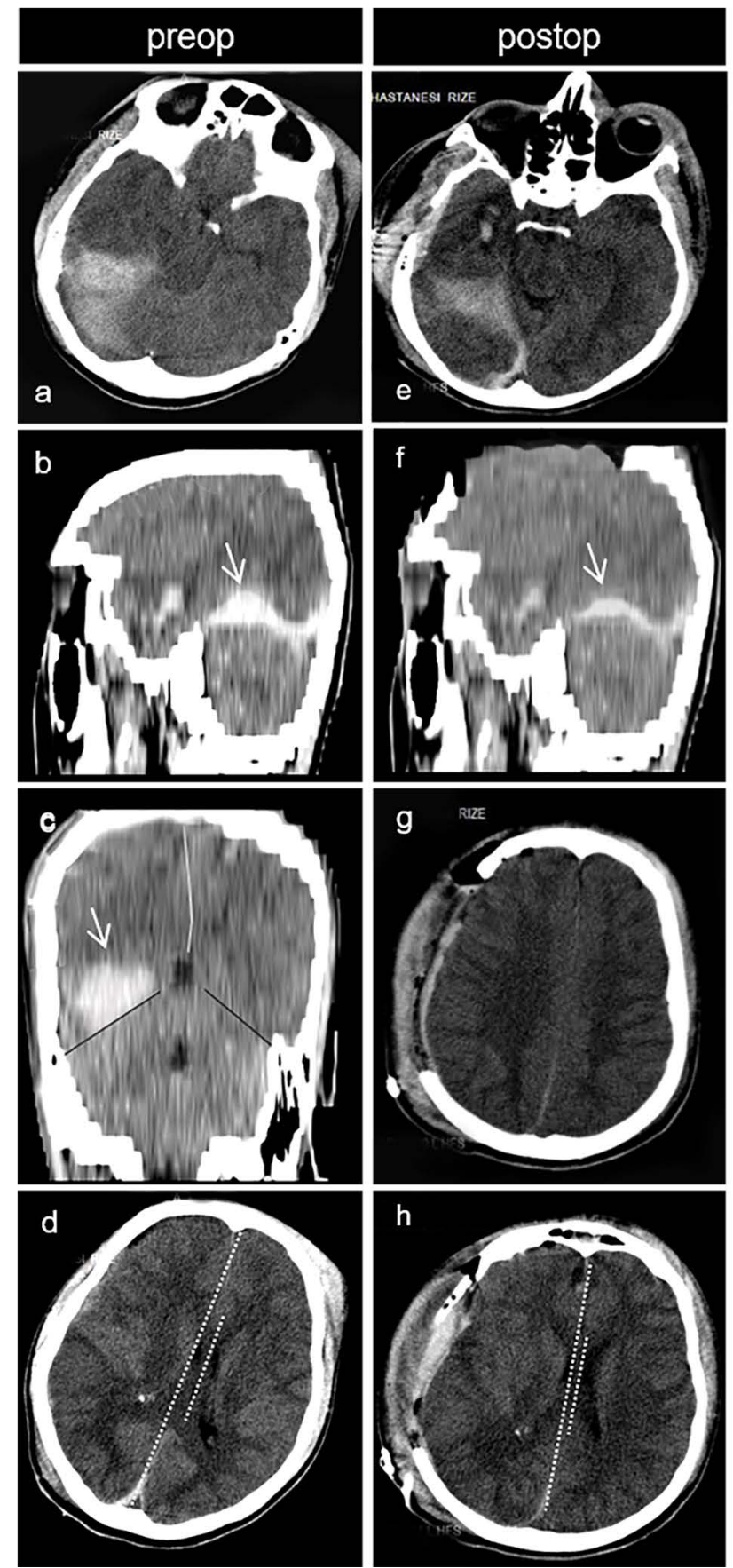

Figure 2 (preoperative images): a) 6 hours later from admission to intensive care of the patient's CT images; b,c) Peritentorial bleeding is observed on axial CT section. Blood volume on sagittal and coronal sections of CT is better understood; d) Midline shift from right to left and minimal subdural hemorrhage on right cerebral hemisphere surface. Postoperative images: Postoperative CT images in 12 Hours; e,f) Compared to the previous section in Figures $2 \mathrm{a}$ and Figure $2 \mathrm{~b}$ are followed by the decreases of the peri-tentorial blood volume and midline shift decreased observed.

to such an appearance on the tentorium, or interhemispheric subdural bleedings that may become diffuse and collected towards the surface of the tentorium [2]. In this case, interhemispheric bleeding was not the subject. It was believed that the bleeding had originated from a large contusion at a deep localization in the left temporal region according to the second radiological evaluation. The literature data reveal that such bleedings from tentorial ruptures may be observed as a result of cranial deformations caused particularly by difficult births. However, the skull of a newborn is far more flexible compared to an adult. The skull of an adult cannot tolerate such a tension and fractures. Such a fracture was not the subject in our case, either.

Bleeding may also be observed as a result of rupture in the tentorial neighboring veins. Anatomically, the bridge veins that are connected to the transverse sinus and the sinuses on the tentorium themselves may be included in these vascular structures. Rosenthal's basal vein variations and the drainage veins of the temporo-occipital lobe are the bridge veins that belong to the tentorium itself. In the study of Matsumoto, et al. [2]. On cadavers, it was reported that the bridge veins on the free edge of the tentorium were larger, similar to (tentorial) venous sinuses, at a rate of $7.6 \%$. Seeger defined the pial and dural penetration points of the veins in the temporo-occipital region. Moreover, it was reported that the variations in the venous system of this region may facilitate APSDH development [1-3].

Peritentorial bleedings may commonly co-exist slightly with other intracranial bleedings in severe head traumas. They may be difficult to identify since they have no major effect on the clinical situation. They may be observed on routine axial brain tomographic images as mild or small bleedings [2,3]. According to the literature data, usually no surgical intervention is necessary $[5,6]$. In the widest series reported in the literature with 32 cases, peritentorial blood was reported to disappear spontaneously on the follow-up of all cases. According to that, this may be considered possible in a pure peritentorial bleeding, but may not be possible in a case with extensive brain edema such as that presented herein. In the presence of an isolated APSDH, drainage may be necessary if the hematoma is large. The radiological images should be carefully evaluated and an intervention may be decided upon, according to the relation with the surrounding structures and poor clinical progression of the hematoma $[2,5]$.

\section{Why did not I choose any other approach to remove the hematoma?}

In this case, the right decision is really important. For example, suboccipital supratentorial approach versus cranial decompressive surgery. I think, is surgery possible direct for cause and how can be it clinical effective? Because of this, decompressive surgery (indirect surgery for that reason) was preferred because of apparent cerebral swelling and low volume of the hematoma in a patient with cerebral herniation. I also know that the patient had little time after the cerebral herniation started.

In the presence of a resistant intracranial hypertension in patients with traumatic excessive cerebral ede- 
ma and damage, decompressive craniectomy may be the decision [3-7]. It may also be the decision in uncal or trans-tentorial herniation situations with a Glasgow Coma Score higher than 3, diffuse cerebral edema and midline shift on brain $\mathrm{CT}$ images, an intra-cerebral pressure of over $40 \mathrm{mmHg}$ within the first 48 hours of trauma [3-5]. Additionally, as direct surgical intervention for the bleeding was impossible, increased bleeding volume is the concern following cranial decompression. However, such a complication was not encountered. Decompressive hemicraniectomy protected the brain stem.

Decreased bleeding volume was detected on the sagittal tomographic sections of the patient, which was obtained following cranial decompression. This was a surprising outcome for us. The relaxation of temporal base has resulted in the dispersion of the peritentorial blood. It has been reported in a very limited number of studies in the literature that removal or reduction of the pressure on the temporal base has resulted in a motion in the subarachnoid space, together with the increased intracranial pressure, and that the cerebrospinal fluid has washed the blood on the tentorial surface and led to its distribution $[7,8]$. The blood on the tentorium may have migrated downward after being collected around the free edges of tentorium in the medial side [8]. The diffuse increase in the intracranial pressure of the case affecting all intracranial areas has prevented further migration of the peritentorial blood downward, and led to an increased pressure on the mesencephaIon. When the herniation was detected, decompression was performed, which mobilized the blood blocked at the level of tentorium post-operatively, although the blood itself was not accessed, and exceeded the tentorial block. Peritentorial blood was confirmed to exceed the tentorial block radiologically following the surgical intervention.

\section{Conclusion}

APSDH usually does not exert a significant mass effect alone. If it co-exist with severe cerebral edema, it may be cause to cerebral herniation. The extensive cra- nial decompression surgery was performed on the skull base. Although it was the indirect surgical approach, Decreased peritentorial bleeding volume was detected following the surgery. This is not the expected result. But, There is proof that how flexible of the brain tissue in a young man. No direct surgical intervention could be performed in the presence of a life-threatening by bleeding in a hard-to-reach area. Although direct surgery for hematoma is not performed, the primary aim is to urgently prevent cerebral herniation. We were thinking that this method for a bleeding in peritentorial localization can be applied.

\section{References}

1. Huang CC, Shen EY (1991) Tentorial subdural hemorrhage in term newborns: Ultrasonographic diagnosis and clinical correlates. Pediatr Neurol 7: 171-177.

2. Matsumoto K, Houri T, Yamaki T, Ueda S (1996) Traumatic Acute Subdural Hematoma Localized on the Superior Surface of the Tentorium Cerebelli; Two Case Reports. Neurol Med Chir (Tokyo) 36: 377-379.

3. Bell RS, Mossop CM, Dirks MS, Stephens FL, Mulligan L, et al. (2010) Early decompressive craniectomy for severe penetrating and closed head injury during wartime. Neurosurg Focus 28: E1.

4. Girotto D, Ledic D, Bajek G, Jerkovic R, Dragicevic S (2011) Efficancy of decompressive craniectomy in treatment of severe brain injury at the Rijeka University Hospital Centre. Coll Antropol 2: 255-258.

5. Koksal V, Oktem IS, Menku A (2009) Acute subdural hematoma in the contralateral side after surgery for chronic subdural hematoma. Erciyes Medical Journal 31: 268-271.

6. Takeuchi S, Takasato Y, Masaoka H, Hayakawa T, Yatsushige $\mathrm{H}$, et al. (2012) Traumatic Peritentorial Subdural Hematomas: A Study of 32 Cases. Turkish Neurosurgery 22: 305-308.

7. Kayaci S, Kanat A, Koksal V, Ozdemir B (2014) Effect of inner membrane tearing in the treatment of adult chronic subdural hematoma: A comparative study. Neurol Med Chir 54: 363-373.

8. Koksal V, Ozdemir B (2015) A Calvarial Acute Subdural Hematoma Migrating into The Spinal Canal in a Young Male: A Case report And Literature Review. Am J Emerg Med 33: 1537 